\title{
Study on Cell-Capturing Microfluidic Device in High Flow Rates through Controlling Shape of Microstructures and Their Alignments ${ }^{\dagger}$
}

\author{
Dae-Sik Lee ${ }^{1, *}$, Jeong Won Park ${ }^{1}$, Moon Youn Jung ${ }^{1}$ and Chunwha Ihm ${ }^{2}$ \\ 1 Biomedical IT Fusion Department, Electronics and Telecommunications Research Institute (ETRI), \\ Daejeon, Korea \\ 2 Department of Laboratory Medicine, Eulji University Hospital, Daejeon, Korea \\ * Correspondence: dslee@etri.re.kr; Tel.: +82-42-860-1543 \\ + Presented at the Eurosensors 2017 Conference, Paris, France, 3-6 September 2017.
}

Published: 22 August 2017

\begin{abstract}
A new cell-trapping method for single cell analysis devices at a very rapid flow rate, is designed, fabricated and characterized by controlling shapes of microstructures and their arrangements in array just simply and effectively within the microfluidic channels. We simulated using a commercialized computational fluidic dynamic simulator (CFD-ACE+) and demonstrated experimentally that the cell-capturing rate enhanced over three times and a high capturing efficiency of about $70 \%$ at a $300 \mu \mathrm{L} / \mathrm{min}$ at the cross-arranged left-turned isosceles trapezoid. The device is applicable for a tool in the field of characterization of rare cells, personal medicine, and prognostic of disease.
\end{abstract}

Keywords: microfluidic device; rare cell capturing; shape control; high flow rate

\section{Introduction}

The introduction should briefly place the study in a broad context and define the purpose of the work and its significance. The handling of single cells is of great importance for cancer research or emerging diagnostic methods [1-3]. Even though some capturing schemes have been reported, passive methods using hydrodynamic weirs seem to be attractive because their implementation is simple and does not need expertise for sophisticated and complex microfluidic control [4]. However, most weir or post systems have relatively poor capturing efficiency, it is needed to enhance the designs and fabrication methods in order to apply for the rare cells populations study [5-7]. Considering the rare cells isolation rate using microfluidic devices is approaching to about $90 \%$, the sequential capturing single cells is also becoming important. Thus, we have presented the a rare cellscapturing device with three different geometries to investigate the effects of geometries on capturing rates in a very high flow rate of $300 \mu \mathrm{L} / \mathrm{min}$, to meet with the condition of over $7.5 \mathrm{~mL} / \mathrm{h}(125 \mu \mathrm{L} / \mathrm{min})$ of a FDA-approved machine [6].

\section{Materials and Methods}

We designed and fabricated a microdevice for the capturing of rare epithelial cancer cells (MCF7, a breast cancer cell line) from human blood based on microshapes and their arrays. A computational simulation using the CFD ACE+ simulator was conducted to observe the lateral flow variations within microfluidic channel due to flow-altering micropatterns to infer the effect on the capturing of rare cells. We have designed various microstructure shapes with three different cross-arranged geometries (rectangle (type 1), right-turned (type 2) or left-turned isosceles trapezoid 
(type 3)) and their gaps for effective single cell (MCF-7, a breast cancer cell line) capturing as shown in Figure 1.

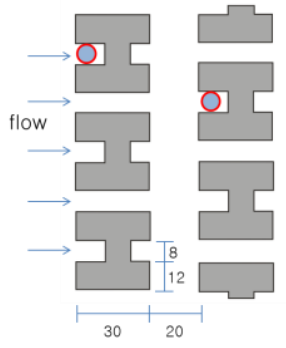

(a)

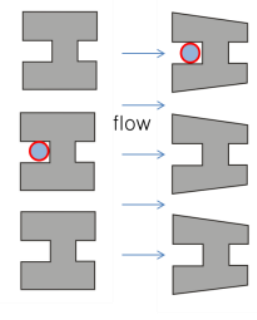

(b)

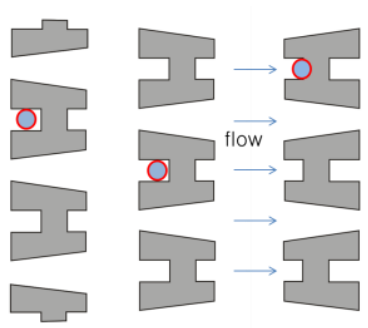

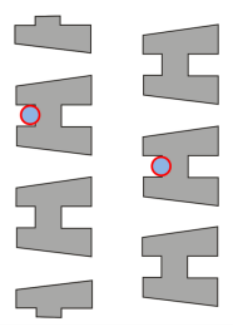

(c)

Figure 1. Three different geometries (type 1: rectangle (a), type 2: right turn trapezoid (b), and type 3: left turn trapezoid (c)) for capturing a breast cancer cell line (MCF-7).

The simulation setup to see the flow rate, a function of distance from the vertically-located posts, is shown in Figure 2. The capture is stimulated by a spatial flow rate variation using a lateral flow module. The fabrication process was designed to be sufficiently simple and suitable for mass-produced protocols. We fabricated the microdevice using clean room facilities of the MEMS Fab center at ETRI. For this, the 6 inch master mold for the three different geometries was fabricated using photolithographic patterning of SU-8, negative tone photoresist, on Si substrate, the microfluidic part was also casted using PDMS (Sylgard 184, Dow Corning Co.) for microfluidic chip. The height of microstructures is about $30 \mu \mathrm{m}$, width in the entrance is about $8 \mu \mathrm{m}$. The cover glass is utilized as a bottom substrate. And the top substrate and bottom substrate have bonded after treatment of oxygen plasma for 1 minute, at $100 \mathrm{sccm}$ flow rate and $100 \mathrm{~mW}$ electric power. To permanently seal the glass bottom plates and a PDMS microfluidic chip with microfluidic channels without the use of conventional adhesives, the PDMS surface was amino functionalized after being oxidized through oxygen plasma treatment. Finally, we have investigated a capturing performance using MCF-7, human breast adenocarcinoma, cells.

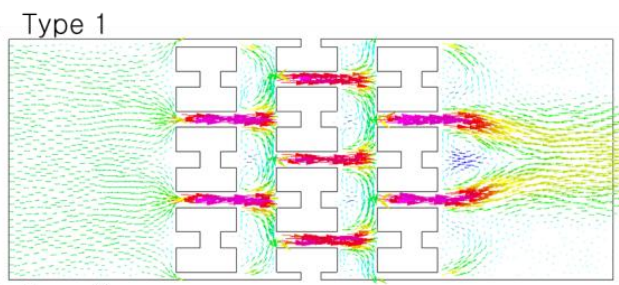

Type 2

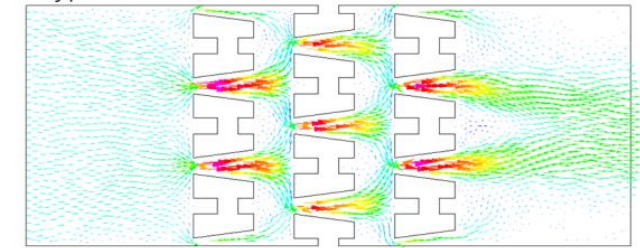

Type 3

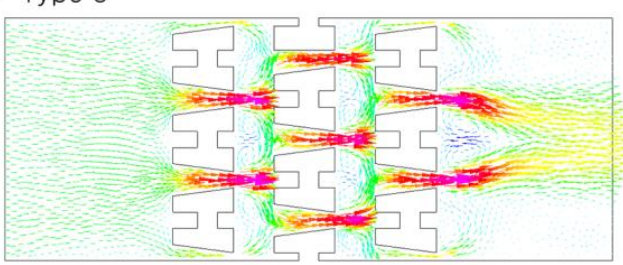

(a)
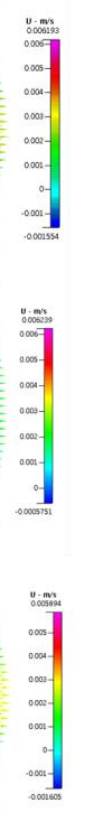
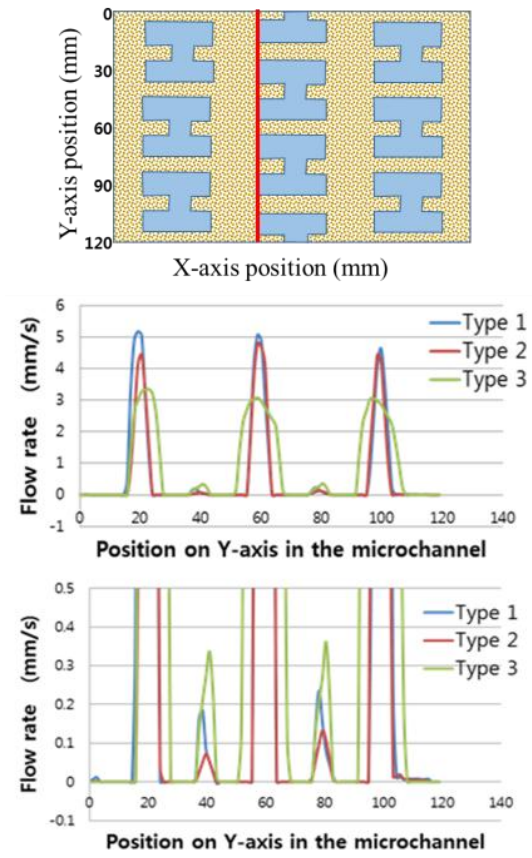

(b)

Figure 2. Microfluidic simulation in three different geometries (type 1: rectangle, type 2: right turn trapezoid, and type 3: left turn trapezoid) for capturing the circulating tumor cells (CTCs) using CFD$\mathrm{ACE}+(\mathbf{a})$, and simulation results of local flow rate variation at entrance of the traps along with red line in three different geometries (type 1, type 2, and type 3) (b). 


\section{Results and Discussion}

The simulation results of flow rate at entrance of the traps along with red line in the geometries for capturing the CTCs are shown in Figure 2. Even though controlling the micro-shapes, there are flow rate changes over three times in the flow rates at the trap parts. Only simple shape change could give the flow rate variation at trap part, resulting in capturing efficiency changes.

The captured CTCs in the device are shown in Figure 3a-c. The capturing efficiency according to the geometry are quite diffent. The capturing efficiency is changed into over three times, dynamically. Thus, geometry design is an important parameter in the capturing efficiency, and the three left-turned trapezoid geometry shows a maximum of about $66 \%$ even at a very fast flow rate of $300 \mu \mathrm{L} / \mathrm{min}$, that is a very harsh condition of $9 \mathrm{~mL}$ for $0.5 \mathrm{~h}$.

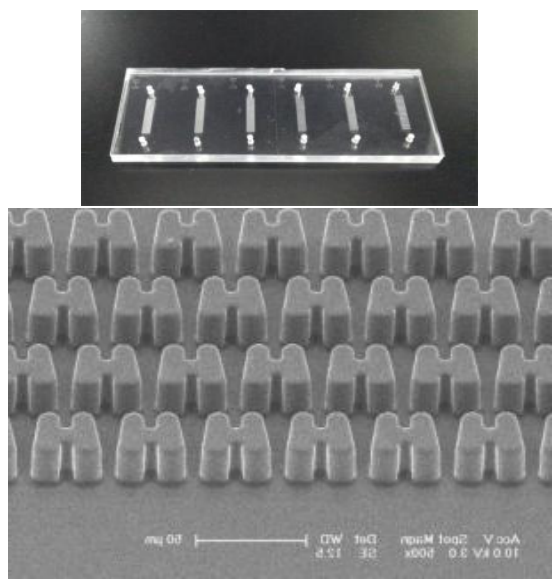

(a)

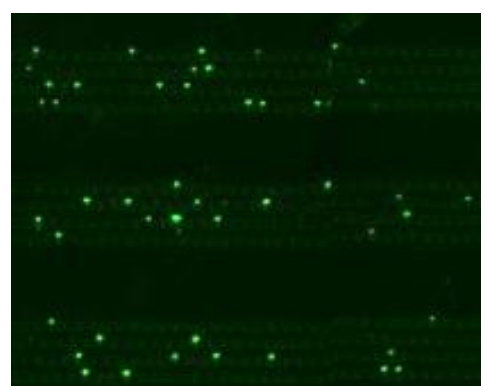

(b)

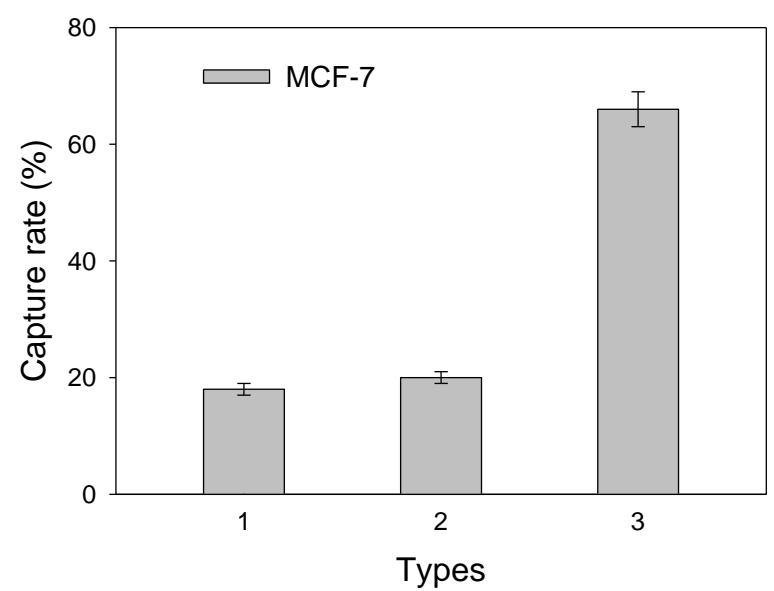

(c)

Figure 3. Photographs of glass-PDMS microfluidic chip, and the magnified SEM image of a crossarranged microstructure (type 3) (a), fluorescence photograph of the captured CTCs (MCF-7) (b), and the capturing rate according to three different geometries (c).

\section{Conclusions}

We have designed and fabricated a PDMS-glass chip using the MEMS processing, the microfluidic part was also casted using PDMS with three different kinds of microstures. Finally, the capturing efficiency difference according to the shape is over three times. We demonstrated that the microdevice showed the good capturing performance at a quite fast flow rate condition and it would be applicable for early cancer detection, personal medicine, and prognosis monitoring of cancer patients in coming future. 
Acknowledgments: This research work was supported by the R\&D Program for Society of the National Research Foundation (NRF) funded by the Ministry of Science, ICT \& Future Planning (Grant number: 2013M3C8A1078454), Republic of Korea.

Conflicts of Interest: The authors declare no conflict of interest. The founding sponsors had no role in the design of the study; in the collection, analyses, or interpretation of data; in the writing of the manuscript, and in the decision to publish the results.

\section{References}

1. Sackmann, E.K.; Fulton, A.I.; Beebe, D.J. The present and future role of microfluidics in biomedical research. Nature 2014, 507, 181-189.

2. Shields, C.W.; Reyes, C.D.; Lopez, G.P. Microfluidic cell sorting: a review of the advances in the separation of cells from debulking to rare cell isolation. Lab Chip 2015, 15, 1230-1249.

3. Carlo, D.D.; Wu, L.Y.; Lee, L.P. Dynamic single cell culture array. Lab Chip 2006, 206, 1445-1449.

4. Choi, D.-H.; Yoon, G.-W.; Park, J.W.; Ihm, C.; Lee, D.-S.; Yoon, J.-B. Fabrication of a membrane filter with controlled pore shape and its application to cell separation and strong single cell trapping. J. Micromech. Microeng. 2015, 11, 105007.

5. Dura, B.; Dougan, S.K.; Barisa, M.; Hoehl, M.M.; Lo, C.T.; Ploegh, H.L.; Voldman, J. Profiling lymphocyte interactions at the single-cell level by microfluidic cell pairing. Nat. Commun. 2015, 6, 5940.

6. Hu, P.; Zhang, W.; Xin, H.; Deng, G. Single, Cell Isolation and Analysis. Front. Cell Dev. Biol. 2016, 4, 116.

7. Park, J.W.; Lee, N.L.; Cho, S.M.; Jung, M.Y.; Ihm, C.; Lee, D.-S. Microdevice for separation of circulating tumor cells using embedded magnetophoresis with $\mathrm{v}$-shaped Ni-Co nanowires and immunonanomagnetic beads. J. Electron. Telecommun. Res. Inst. ETRI 2015, 37, 233-240.

(C) 2017 by the authors. Licensee MDPI, Basel, Switzerland. This article is an open access article distributed under the terms and conditions of the Creative Commons Attribution (CC BY) license (http://creativecommons.org/licenses/by/4.0/), 